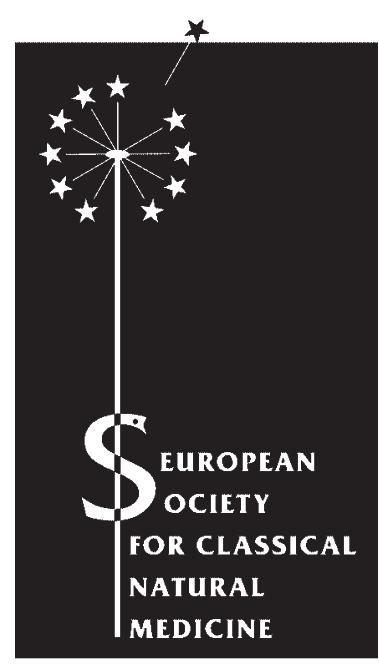

Gesellschaftsmitteilungen - Society Bulletins

Forsch Komplementärmed Klass Naturheilkd 2004;11:314-315

\section{Zusammenfassung des ESCNM-Kongresses in Zaragoza, Spanien, 7. bis 9. Mai 2004}

Der Kongress für naturheilkundliche Medizin fand in der Medizinfakultät der Universität von Zaragoza statt und war dem Herzen gewidmet. Die Kongressteilnehmer kamen aus vielen europäischen Ländern sowie aus Lateinamerika, unter anderem aus Deutschland, Spanien, Peru, Venezuela, Mexiko und Kuba. Der nachfolgende Beitrag fasst die wichtigsten Schlussfolgerungen dieses Kongresses für die europäischen Gesundheitspolitik zusammen.

Eröffnet wurde der Kongress durch den Präsidenten des Ehrenkomitees und Pathologen, Dr. Jesús Lázaro Pérez, der in seinem einführenden Vortrag auch von den besonderen Gefühlen berichtete, ein Herz in den Händen zu halten - Gefühle, die sich bei anderen Organen so nicht einstellen.

Der Kongress war in 6 Diskussionsforen unterteilt:

\section{Gesundheitsgewohnheiten, Koordinator:}

\section{José Rafael Rodriguez Sanchez-Bretaño}

Dr. Dieter Melchart, Leiter des Projekts zur Selbstverwaltung und -überprüfung der Gesundheit vom Patienten, berichtete von der Wichtigkeit dieser Methode bei Herzkrankheiten. Dass der Patient seine Gesundheit überprüft, in Selbsthilfegruppen mehr über die Krankheit lernt, seine Laborwerte besser bewerten kann und mögliche Therapieansätze vorschlägt - natürlich immer in Rücksprache mit dem Arzt/Ärztin -, ist für den Verlauf der Erkrankung von grosser Bedeutung. Ergänzend werden in diesem Projekt auch Kurse zur Verbesserung des Lebensstils angeboten.

Dr. Milagros Bernal Pérez sprach über die grossen Vorteile des Verzichts auf Tabakkonsum, vor allem hinsichtlich der Vorbeugung von Herzkrankheiten. Besonders betonte sie die hohe gesellschaftliche Anerkennung für diesen Verzicht.

Dr. Andreas Michalsen wies darauf hin, wie hoch in den medizinischen Publikationen, insbesondere in denen zur Vorbeugung von Herzkrankheiten, der Anteil von unterschiedlichen Techniken der klassischen Naturheilkunde ist. Eine ausgewo- gene Ernährung, Bewegung, Hydrotherapie und Entspannungstechniken sollten die Basis für eine Primär-, aber auch Sekundär- und Tertiärprophylaxe bei Herzkrankheiten sein.

\section{Geschichte, Volksmedizin und Anthroposophie, Koordinator: José Maria Poveda}

Dr. Francisco Verdú berichtete in seinem Vortrag «Herz und Geschichte. Miguel SeNet, ein Wegbereiter» über die Historie und die Wissensquellen von SeNet, die in gleicher Weise in verschiedenen Kulturen, etwa der ägyptischen, indischen, arabischen, jüdischen und griechischen, zu finden sind. Er sprach auch über das Wissen, das all diese Kulturen zur Physiologie und Funktion des Herzens beigetragen haben - eine gute $\mathrm{Zu}$ sammenfassung von zirka 3000 Jahren in 20 Minuten.

Dr. Carmen Flores fasste die Ansichten der Anthroposophie über das Herz zusammen - eine Mischung aus Wissenschaft und Poesie mit einer überwältigenden Logik, die das Physische mit dem Psychischen und Spirituellen vereint.

Dr. José Maria Poveda berichtete über die Bedeutung der schamanischen Kulturen und ihrer einfachen Techniken zur Pflege des Herzens, die ihren Ursprung in der Beobachtung der Natur und des Tierreichs haben.

\section{Psychoneuroimmunologie, Koordinator: \\ Rómulo Rodriguez Ramos}

Dr. Judit Rodriguez wies in ihrem Vortrag auf die engen Beziehungen zwischen Herz und Immunsystem hin, insbesondere auf die über weite Bereiche identischen Risikofaktoren. Es besteht eine enge Korrelation zwischen Erkrankungen des Immunsystems und koronaren und Gefässerkrankungen.

Dr. Oscar Villavicencio sprach über die Verbindung der allgemeinen Gesundheit mit der des Herzens. Er betonte die Bedeutung der Fähigkeit des Arztes, auf seine Patienten einzugehen. Die Vorbildfunktion des Arztes in Bezug auf gesundheitsfördernde Lebensgewohnheiten sind Teil der Therapie und haben einen grossen Einfluss auf deren Erfolg.

Dr. Rómulo Rodriguez Ramos betonte die Tatsache, dass die Psychoneuroimmunologie kein Phantasiegebilde ist, sondern eine Wissenschaft, die präzise vorweisen kann, welch starken
KARGER

Fax +497614520714

(ㅇ) 2004 S. Karger GmbH, Freiburg

Accessible online at:

rmation@Karger.de www.karger.com/fkm

www.karger.com 
Einfluss das psychologische Verhalten jedes Individuums auf seine Gesundheit und die Gesundheit des Herz-KreislaufSystems hat.

Shravani S. Pawar berichtete von seiner Arbeit und der Ausübung von Yoga als ganzheitlicher Vorsorgemassnahme sowie zur Behandlung von Herzkrankheiten. Er beendete seinen Vortrag mit einem Mantragesang, der alle Herzen der Aula Magna schlagen liess.

\section{Diagnostik, Koordinator: Isidro Blasco}

Dr. Max H. Pittler sprach über die aktuellen Überprüfungen in der Medizin und über die Ergebnisse des «Cochrane-Instituts», die vor allem Studien zum Einsatz von Heilpflanzen bestätigen. Weiter wies er auf die hohe Effizienz der Therapie mit bestimmten Pflanzen hin, auch wenn diese noch nicht mit Studien belegt worden ist.

Dr. Luis Javier Placer referierte über neue Perspektiven in der Diagnostik von Herz-Kreislauf-Erkrankungen und wies darauf hin, dass in Verbindung mit den bereits bekannten Diagnostikmethoden ein preisgünstiges Instrumentarium zur Erkennung der bestehenden und fortgeschrittenen Krankheit, aber auch zur Vorbeugung vorliegt. Wie sich zeigt, ist die Änderung der Lebensgewohnheiten das Einzige, was der Entstehung von Herz-Kreislauf-Krankheiten vorbeugen kann.

\section{TCM}

Alfredo Embid analysierte 46 randomisierte Studien und eine Akupunktur-Kontrollgruppe mit den folgenden Krankheiten: Herzinsuffizienz, koronare Herzkrankheiten, Herzrhythmusstörungen und Bluthochdruck.

Dr. Carlos Pomarón sprach über die Bedeutung eines einzigen Akupunkturpunkts, Neiguan (6PC) und dessen Auswirkung auf das sympathische System.

Dr. Francisco Abad-Alegría wies auf die beginnenden Aspekte der koronartherapeutischen Akupunktur hin und motivierte zur weiteren Forschung in diesem Feld.

\section{Naturheilkundliche Behandlungen}

Dr. Francisco Maraver wies auf den grossen Erfahrungsschatz der Balneotherapie hin und stellte die Kurbäder für HerzKreislauf-Erkrankungen und deren Therapien und genaue Anwendungen vor.
Dr. Rainer Stange legte nahe, dass Methoden der Naturheilkunde eine grosse therapeutische Bedeutung für die Behandlung von Herzinsuffizienz haben. Patienten mit dieser Krankheit, die nur mit Medikamenten behandelt werden, zeigen eine progressive Krankheitsverschlechterung, die durch die richtige Anwendung von Bewegungs-, Ernährungs-, Hydround Meditationstherapien einfach zu vermeiden wäre. Diese naturheilkundlichen Therapien verbessern nicht nur die Lebensqualität des Patienten, sondern wirken sich auch positiv auf den Verlauf der Krankheit aus.

Dr. José Luis Berdoncés fasste die aktuellen Therapieformen der Phytotherapie bei koronaren Krankheiten zusammen Medikamente, Heilpflanzen und Ernährung. Er wies auf die Bedeutung und die Rolle jeder einzelnen dieser Therapien hin.

Zum Abschluss des Kongresses wurden folgende Ziele formuliert, zu deren Erreichung von Seiten der ESCNM auch eine Zusammenarbeit mit europäischen Regierungen angestrebt wird:

- Verbreitung und Bekanntmachung der klassischen Naturheilkunde

- Unterstützung von Doktorarbeiten im Bereich der Forschung und Lehre der Naturheilkunde

- Durchführung von zweisprachigen (Englisch und Sprache des Veranstaltungsorts) Sommerkursen in Kooperation mit europäischen Universitäten

- Aufbau einer Zusammenarbeit mit Lateinamerika, um zukünftig Projekte durchzuführen.

Prof. Dr. med. Pablo Saz, Zaragoza

\section{Weitere Informationen}

Die Sommer School findet 2004 leider nicht statt.

\section{Vorankündigung}

Die Vorstandssitzung findet Ende September 2004 in München statt.

7. Summer School für Klassische Naturheilkunde: 23. September bis 03. Oktober 2005. Weitere Informationen unter www.summer-school.de 